\title{
THE COHOMOLOGY GROUPS OF TORI IN FINITE GALOIS EXTENSIONS OF NUMBER FIELDS
}

\author{
J. TATE
}

To the memory of TADASI Nakayama

\begin{abstract}
Class field theory determines in a well-known way the higher dimensional cohomology groups of the idèles and idèle classes in finite Galois extensions of number fields. At the Amsterdam Congress in 1954 I announced [7] the corresponding result for the multiplicative group of the number field itself, but the proof has never been published. Meanwhile, Nakayama showed that results of this type have much broader implications than had been realized. In particular, his theorem allows us to generalize our result from the multiplicative group to the case of an arbitrary torus which is split by the given Galois extension. We also treat the case of "S-units" of the multiplicative group or torus, for a suitably large set of places $S$. It is a pleasure for me to publish this paper here, in recognition of Nakayama's important contributions to our knowledge of the cohomological aspects of class field theory; his work both foreshadowed and generalized the theorem under discussion.
\end{abstract}

Notations, and the plan of the proof. Let $L$ be an algebraic number field of finite degree, or an algebraic function field of one variable over a finite constant field, and let $K / L$ be a finite Galois extension with group $G$. By a place of $K$ we mean an equivalence class of non-trivial absolute values, archimedean or non-archimedean. Let $S$ be a set of places of $K$ satisfying the following conditions :

(S1) $S$ is stable under $G$.

(S2) $S$ contains all archimedean places.

(S3) $S$ contains all places ramified over $L$.

(S4) $S$ is large enough so that every ideal class of $K$ contains an ideal with support in $S$.

Received September 20, 1965. 
Note that we do not assume that $S$ is finite, and that the conditions are automatically satisfied if $S$ is the set of all primes of $K$, a case which is certainly of interest in what follows.

We wish to study the Galois cohomology of the following exact sequence of $G$-modules

$$
0 \rightarrow E \stackrel{a^{\prime}}{\longrightarrow} J \stackrel{a}{\longrightarrow} C \longrightarrow 0
$$

in which:

$E$ is the group of $S$-units of $K$, that is, elements of $K$ which are units at all places $P$ not in $S$.

$J$ is the group of $S$-idèles of $K$, that is, idèles whose $P$-component is a unit for each place $P$ not in $S$.

$C$ is the group of $S$-idèle classes, which in view of condition (S4) is isomorphic to the group of all idèle classes of $K$.

We will compare the cohomology of (A) with that of a simpler sequence, which we denote by

$$
0 \longrightarrow X \stackrel{b^{\prime}}{\rightarrow} Y \stackrel{b}{\rightarrow} Z \longrightarrow 0
$$

\section{Here :}

$Z$ is the group of integers, $G$ operating trivially.

$Y$ is the free abelian group generated by the places $P$ in $S$, an element $s \in G$ operating by the rule

$$
s\left(\sum_{P \in S} n_{P} P\right)=\sum_{P \in S} n_{P}(s P)=\sum_{P \in S} n_{\left(S^{-1} P\right.} P .
$$

$X$ is the kernel of the map $b$ which takes an element

$$
y=\sum n_{P} P \text { into its coefficient sum, } \sum n_{P} .
$$

Clearly $X$ is a $G$-submodule of $Y$, and (B) is an exact sequence of left $G$ modules. Perhaps we should make precise that we are letting $G$ act on places of $K$ according to the convention $|c|_{s P}=\left|s^{-1} c\right|_{P}$, for $c \in K$, where $c \mapsto|c|_{P}$ is an absolute value belonging to the place $P$; thus the rule $s(t P)=(s t) P$ holds for $s, t \in G$.

Our object is to show that the cohomology sequence derived from (A) is isomorphic to that derived from (B), after a dimension shift of two; that is, 
we want to construct a commutative diagram

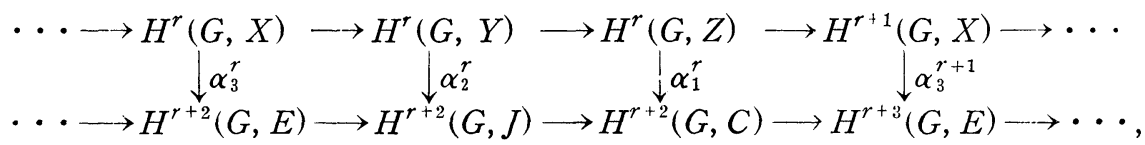

in which the vertical arrows $\alpha_{i}^{r}$, for $i=1,2,3$ and $-\infty<r<\infty$, are isomorphisms. Here, and throughout this paper, $H^{r}(G, M)$ denotes the modified cohomology groups of the finite group $G$ operating on the $G$-module $M$, which are in general non-trivial in negative dimensions, and which are denoted by $\hat{H}^{r}$ in [2, Ch. 12] and in [5, Chs. 8 and 9], where their properties are discussed.

The homomorphisms $\alpha_{i}^{r}$ will be given by cup product with certain cohomology classes

(2) $\alpha_{3} \in H^{2}(G, \operatorname{Hom}(X, E)), \alpha_{2} \in H^{2}(G, \operatorname{Hom}(Y, J))$, and $\alpha_{1} \in H^{2}(G, \operatorname{Hom}(Z, C))$ which we are soon going to define. Here and throughout this paper, "Hom" denotes abelian group homomorphisms; we use "Hom ${ }_{G}$ " to denote $G$-module homomorphisms. If $M$ and $N$ are $G$-modules, then we make $\operatorname{Hom}(M, N)$ into a $G$-module by putting $(s f)(x)=s\left(f\left(s^{-1} x\right)\right)$ for $s \in G, f \in \operatorname{Hom}(M, N)$, and $x \in M$. Then the rule $(s f)(s x)=s(f(x))$ holds, so that the canonical pairing

$$
\operatorname{Hom}(M, N) \times M \rightarrow N
$$

is a $G$-pairing and does lead to cup products

$$
H^{s}(G, \operatorname{Hom}(M, N)) \times H^{r}(G, M) \longrightarrow H^{s+r}(G, N) .
$$

Thus, any three cohomology classes $\alpha_{i}$ as in (2) will lead to a diagram (1) in which $\alpha_{i}^{r}(\xi)=\alpha_{i} \cup \xi$.

In order that the diagram (1) be commutative it suffices that the three classes $\alpha_{i}$ be the images of one single canonical class

$$
\alpha \in H^{2}(G, \operatorname{Hom}((\mathrm{B}),(\mathrm{A}))),
$$

under the three maps $u_{i}$ in the following commutative diagram

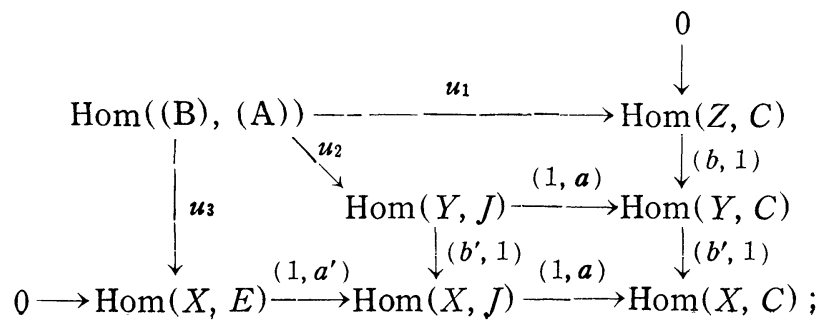


here $\operatorname{Hom}((\mathrm{B}),(\mathrm{A}))$ is the group of triplets $f=\left(f_{1}, f_{2}, f_{3}\right)$ of homomorphisms $f_{i}$ of abelian groups such that the following diagram is commutative

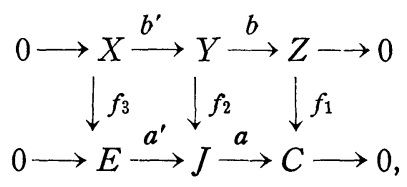

and the homomorphisms $u_{i}$ are defined by $u_{i}(f)=f_{i}$ for $i=1,2,3$. An arrow in (5) marked $(v, w)$ carries a homomorphism $f$ into $w \circ f \circ v$. If $\alpha$ is any class as in (4) and we put $\alpha_{i}=u_{i}(\alpha)$ then we have

$$
\alpha_{i}^{r}(\xi)=\alpha_{i} \cup \xi=\alpha \cup \xi, \quad \text { for } i=1,2 \text {, and } 3 \text {, }
$$

and the commutativity of the resulting diagram (1) results from the basic functorial properties of the cup product; the point is that the single group $\operatorname{Hom}((B),(A))$ is paired compatibly with the whole sequence $(B)$ into the sequence $(A)$.

The method by which we will construct $\alpha$ is as follows. From global (resp. local) class field theory we get first a canonical class $\alpha_{1}$ (resp. $\alpha_{2}$ ) as in (2) such that the arrows $\alpha_{1}^{r}$ (resp. $\alpha_{2}^{r}$ ) in (1) are isomorphisms for all $r$. Then, using the relationship between the local and global class field theories we show that there exists a unique class $\alpha$ as in (4) such that $\alpha_{i}=u_{i}(\alpha)$ for $i=1,2$. Putting $\alpha_{3}=u_{3}(\alpha)$ we finally obtain a commutative diagram (1) in which, by the five lemma, all vertical arrows are isomorphisms.

Construction of $\alpha_{1}$. We let $\alpha_{1}$ be the image under the canonical isomorphism $C \simeq \operatorname{Hom}(Z, C)$ of the fundamental class in $H^{2}(G, C)$; cf. [1], [3]. It is well known that the maps $\alpha_{1}^{r}: H^{r}(G, Z) \rightarrow H^{r+2}(G, C)$ produced by cupping with $\alpha_{1}$ are isomorphisms for all $r$; cf. [4], [5], [6].

Construction of $\alpha_{2}$. Let us first recall some facts about the functors $M$ $H^{r}(G, M)$ which we will use. Since these functors can be viewed as cohomology, they commute with direct products; but since they can be viewed equally well as homology, they commute with direct sums and injective limits as well. Shapiro's lemma, which relates the cohomology of a group to that of a subgroup, can be stated as follows: Let $G$ be a finite group, $G_{1}$ a subgroup and $G=\cup_{t \in r} t G_{1}$ a partition of $G$ into cosets of $G_{1}$, Let $M$ be a $G$-module, $M_{1}$ a 
$G_{1}$-module, and

$$
M \underset{i}{\stackrel{j}{\rightleftarrows}} M_{1}
$$

a pair of $G_{1}$-homomorphisms such that $j \circ i$ is the identity on $M_{1}$ and such that $M=\sum_{t \in T} t i M_{1}$, direct sum. Then the two maps

$$
H^{r}(G, M) \underset{\text { cor } \circ i}{\stackrel{j \circ \mathrm{res}}{\rightleftarrows}} H^{r}\left(G_{1}, M_{1}\right)
$$

are mutually inverse isomorphisms, where res denotes restriction and cor denotes "corestriction" or "transfer". The fundamental relation between transfer and cup products is

$$
\alpha \cup \operatorname{cor} \beta=\operatorname{cor}(\operatorname{res} \alpha \cup \beta),
$$

which holds for any $G$-pairing and any subgroup $G_{1}$ of $G$, where $\alpha$ is a $G$ cohomology class in the first factor of the pairing and $\beta$ a $G_{1}$-cohomology class in the second. Finally we note that we are systematically using the same letter to denote the induced homomorphism on cohomology as denotes the $G$ homomorphism by which it is induced.

For each place $P$ of $K$, let $G_{P}$ denote the corresponding decomposition group, that is, the group of all $s \in G$ such that $s P=P$, and let $K_{P}$ denote the completion of $K$ with respect to the place $P$. For each $P$ in $S$, let

$$
Z \stackrel{i^{\prime} P}{\rightarrow} Y \text { and } K_{P}^{*} \stackrel{i_{P}}{\longrightarrow} J
$$

denote the canonical injections, the first of which is defined by $i_{P}^{\prime}(n)=n P$, and the second of which maps a non-zero element of $K_{P}$ onto the idèle having that element as $P$-component and having 1 as its component at all places other than $P$. Then $i_{P}^{\prime}$ and $i_{P}$ are $G_{P}$-homomorphisms and so also, for any $G$-module $M$, is the projection

$$
j_{P}: \operatorname{Hom}(Y, M) \rightarrow M
$$

defined, for $P$ in $S$, by $j_{P}(f)=f(P)$.

Let $S_{*}$ denote the set of places of $L$ which lie below the places in $S$, and let $h: S_{*} \rightarrow S$ be a function which assigns to each place $Q$ in $S_{*}$ a place $P$ lying over $Q$. 
Lemma. For any G-module $M$ there is a canonical isomorphism

$$
H^{r}(G, \operatorname{Hom}(Y, M)) \simeq \prod_{Q \in S_{*}} H^{r}\left(G_{h(Q)}, M\right)
$$

which attaches to an element $\theta$ on the left the element of the direct product whose $Q$-component is $j_{h(Q)} \circ \operatorname{res}(\theta)$ for each $Q \in S_{*}$.

Note that $Y$ is the direct sum of the $G$-modules $Y_{Q}=\sum_{\left.P\right|_{Q}} Z P$. Accordingly, we have

$$
\operatorname{Hom}(Y, M) \simeq \prod_{Q} \operatorname{Hom}\left(Y_{Q}, M\right) .
$$

Now recall that cohomology commutes with products, and apply Shapiro's lemma to the situation

$$
\operatorname{Hom}\left(Y_{Q}, M\right) \underset{i}{\stackrel{j}{\rightleftarrows}} M,
$$

where $j(F)=f(h(Q))$ and where $(i(x))\left(\sum n_{P} P\right)=n_{h(Q)} x$ for $x \in M$. Shapiro's lemma applies, because any two primes of $K$ lying over the same prime of $L$ are in the same orbit under $G$.

In view of this lemma we can define $\alpha_{2}$ to be the unique element of $H^{2}(G, \operatorname{Hom}(Y, J))$ such that, for each $Q \in S_{*}$ we have

$$
j_{h(Q)}\left(\operatorname{res} \alpha_{2}\right)=i_{h(Q)} \alpha_{h(Q)} \quad \text { in } H^{2}\left(G_{h(Q)}, J\right),
$$

where for each place $P$ of $K$ we let $\alpha_{P}$ denote the local fundamental class in $H^{2}\left(G_{P}, K_{P}^{*}\right)$.

The class $\alpha_{2}$ defined by (8) is independent of the choice of the function $h$; indeed we have

$$
j_{P}\left(\text { res } \alpha_{2}\right)=i_{P} \alpha_{P} \quad \text { in } H^{2}\left(G_{P}, J\right)
$$

for every place $P$ in $S$. Let $P=t h(Q)$, with $t \in G$. The automorphism $t$ acts on the whole situation under consideration, which is derived functorially from the field $K$. If we apply $t$ to (8) we obtain

$$
j_{P} \operatorname{res}\left(t_{*} \alpha_{2}\right)=i_{P}\left(t_{*} \alpha_{h(Q)}\right)
$$

where $t_{*} \alpha_{2}$ is the image of $\alpha_{2}$ by transport of structure under the automorphism $(G, J) \rightarrow(G, J)$ defined by $(s, x) \quad\left(t s t^{-1}, t x\right)$ and where $t_{*} \alpha_{P}$ is the image of $\alpha_{h(Q)}$ by transport of structure under the isomorphișm $\left(G_{h(Q)}, K_{h(Q)}^{*}\right) \rightarrow\left(G_{P}, K_{P}^{*}\right)$ 
defined similarly. But it is a well-known fact about the cohomology of groups that $t_{*} \alpha_{2}=\alpha_{2}$, and we have $t^{*} \alpha_{h(\varphi)}=\alpha_{P}$ because the fundamental class of local class field theory is canonical. Formula $\left(8^{\prime}\right)$ results.

Proof that $\alpha_{2}^{r}$ is an isomorphism. We shall prove more, namely that the following diagram is commutative.

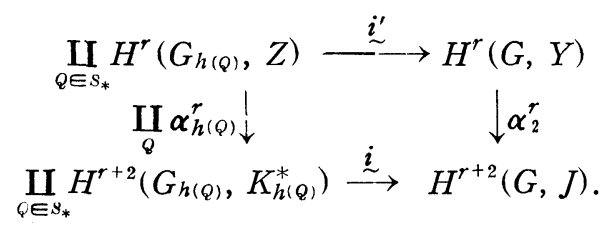

The sign II denotes direct sum. The left hand vertical arrow is an isomorphism because for each place $P$ of $K$ the cup product with $\alpha_{P}$ gives an isomorphism $\alpha_{P}^{r}: H^{r}\left(G_{P}, Z\right) \underset{\sim}{\longrightarrow} H^{r+2}\left(G_{P}, K_{P}^{*}\right)$, cf. [4], [5], [6]. The top horizontal arrow $i^{\prime}$ is induced by the maps

$$
H^{r}\left(G_{P}, Z\right) \stackrel{\text { coro } i^{\prime} P}{\longrightarrow} H^{r}(G, Y),
$$

for $P=h(Q)$; it is an isomorphism because $Y$ is the direct sum of the $Y_{Q}$, and Shapiro's lemma applies to the situation $i_{P}^{\prime}: Z \rightarrow Y_{Q}$. The bottom horizontal arrow $i$ is induced by the maps

$$
H^{r}\left(G_{P}, K_{P}^{*}\right) \stackrel{\operatorname{cor}^{\circ} i_{P}}{\longrightarrow} H^{r}(G, J),
$$

for $P=h(Q)$, and is well known to be an isomorphism. For completeness we sketch the proof. We have

$$
J=\underset{s_{0}}{\lim } J\left(S_{0}\right)
$$

where $S_{0}$ runs over the family of finite subsets of $S_{*}$, ordered under inclusion, and where $J\left(S_{0}\right)$ is the group of idèles whose components are units at the places $P$ above $S_{0}$; that is,

$$
J\left(S_{0}\right)=\prod_{Q \in \rho_{0}} J_{Q} \times \prod_{Q \nsubseteq S_{0}} U_{Q}
$$

where

$$
J_{Q}=\prod_{P \mid Q} K_{P}^{*} \text {, and } U_{Q}=\prod_{P \mid Q} U_{P},
$$

$U_{P}$ denoting the group of units in $K_{P}$. Now use the commutativity of our 
cohomology with products and injective limits, apply Shapiro's lemma to $J_{Q}$ and $U_{Q}$, and recall that $H^{r}\left(G_{P}, U_{P}\right)=0$ for all $r$ when $K_{P} / L_{Q}$ is unramified, which is the case for all but a finite number of places $Q$ of $L$.

To prove the commutativity of (9) it suffices to show

$$
\alpha_{2} \cup\left(\operatorname{cor} i_{F}^{\prime} \xi\right)=\operatorname{cor} i_{P}\left(\alpha_{P} \cup \xi\right)
$$

for each $P=h(Q)$ and each $\xi \in H^{r}\left(G_{P}, Z\right)$. By (7) the left side is equal to $\operatorname{cor}\left(\operatorname{res} \alpha_{2} \cup i_{P}^{\prime} \xi\right)$, and we have

$$
\text { res } \alpha_{2} \cup i_{P}^{\prime} \xi=\left(j_{P} \operatorname{res} \alpha_{2}\right) \cup \xi=\left(i_{P} \alpha_{P}\right) \cup \xi=i_{P}\left(\alpha_{P} \cup \xi\right) .
$$

The two extreme equalities come from the functorality of cup products and the middle one from $\left(8^{\prime}\right)$.

Construction of $\alpha$. From the upper part of diagram (5) we obtain a sequence

$$
0 \longrightarrow \operatorname{Hom}((\mathrm{B}),(\mathrm{A})) \stackrel{\left(\boldsymbol{u}_{1}, u_{2}\right)}{\longrightarrow} \operatorname{Hom}(Z, C) \times \operatorname{Hom}(Y, J) \stackrel{\left(\begin{array}{l}
(b, 1) \\
-(1, a)
\end{array}\right)}{\longrightarrow} \operatorname{Hom}(Y, C) \longrightarrow 0
$$

which is easily seen to be exact. On the left and in the middle the exactness follows formally from the definition of $\operatorname{Hom}(\mathrm{B}),(\mathrm{A}))$ and the exactness of (5). At the right the exactness results from the fact that $Y$ is $Z$-free, so that the homomorphism $(1, a): \operatorname{Hom}(Y, J) \rightarrow \operatorname{Hom}(Y, C)$ is surjective. Now consider the exact cohomology sequence derived from (10). In order to show that there is an $\alpha \in H^{2}(G, \operatorname{Hom}((\mathrm{B}),(\mathrm{A})))$ such that $u_{1}(\alpha)=\alpha_{1}$ and $u_{2}(\alpha)=\alpha_{2}$ we have only to show that

$$
(b, 1) \alpha_{1}=(1, a) \alpha_{2}, \quad \text { in } H^{2}(G, \operatorname{Hom}(Y, C)) .
$$

Moreover such an $\alpha$ is unique if it exists, because by our lemma,

$$
H^{1}(G, \operatorname{Hom}(Y, C)) \simeq \prod_{Q \in s_{*}} H^{1}\left(G_{h(Q)}, C\right)=0 .
$$

By the same lemma, to check (11) it suffices to check

$$
j_{P} \operatorname{res}(b, 1) \alpha_{1}=j_{P} \operatorname{res}(1, a) \alpha_{2}, \quad \text { in } H^{2}\left(G_{P}, C\right)
$$

for each place $P \in S$. This boils down to showing

$$
\operatorname{res} \alpha_{1}=a\left(j_{P} \text { res } \alpha_{2}\right), \quad \text { in } H^{2}\left(G_{P}, C\right),
$$


which is a problem concerning the relation between local and global fundamental classes in the extension $K / L^{\prime}$ whose Galois group is $G_{P}$. Let $Q^{\prime}$ denote the place of $L^{\prime}$ below $P$. The local degree of $K / L^{\prime}$ at $Q^{\prime}$ is equal to the global degree, $n^{\prime}=\left[K: L^{\prime}\right]$, and $P$ is the only place of $K$ above $Q^{\prime}$. Since restriction carries fundamental class into fundamental class, the left side of (12) is the global fundamental class for $K / L^{\prime}$, and has therefore the invariant $1 / n^{\prime}$. On the other hand, by the definition of $\alpha_{2}$, the class $j_{P}$ res $\alpha_{2}$ in $H^{2}\left(G_{P}, J\right)$ has invariant $1 / n^{\prime}$ at $Q^{\prime}$ and 0 at all other places of $L^{\prime}$. The sum of these invariants, namely $1 / n^{\prime}$, is the invariant of the right side of (12); hence (12) is true.

The generalization to tori. Let $M$ be a torsion free $G$-module. Let $(\mathrm{A}) \otimes M($ resp. $(\mathrm{B}) \otimes M)$ denote the result of tensoring the exact sequence (A) (resp. (B)) with $M$, over $Z$. Since $M$ is torsion free, these new sequences are exact. We let $G$ operate on them by the rule $s(x \otimes y)=s x \otimes s y$. Then the obvious pairing

$$
\operatorname{Hom}((\mathrm{B}),(\mathrm{A})) \times((\mathrm{B}) \otimes M) \rightarrow(\mathrm{A}) \otimes M
$$

is a $G$-pairing, and the cup product with the canonical class $\alpha$ just constructed gives the vertical arrows in a commutative diagram

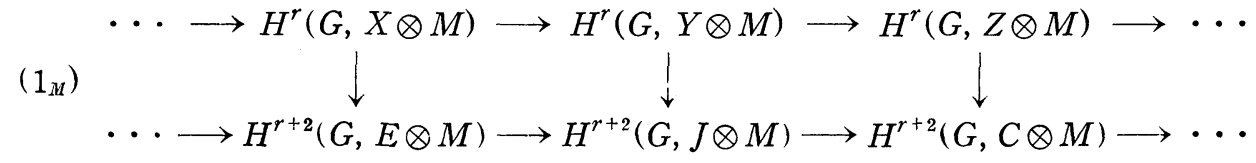

analogous to $(1)=\left(1_{z}\right)$.

TheOREM. The vertical arrows in $\left(1_{M}\right)$ are isomorphisms.

By Nakayama's theorem [4], [5] we have only to check that they are isomorphisms when $M=Z$, but with $G$ replaced by an arbitrary subgroup $G^{\prime}$ of $G$. This follows from what we have proved, together with

Proposition. Let $G^{\prime}$ be a subgroup of $G$. The canonical class $\alpha^{\prime} \in H^{2}\left(G^{\prime}, \operatorname{Hom}((\mathrm{B}),(\mathrm{A}))\right)$ is the restriction of $\alpha$.

By the definition of $\alpha$ and $\alpha^{\prime}$ via the exact sequence (10), it suffices to prove the proposition with $\alpha$ replaced by $\alpha_{1}$ and $\alpha_{2}$. For $\alpha_{1}$, this is just the basic property of global fundamental classes to be carried into each other by 
restriction. To check the statement for $\alpha_{2}$, we let the symbols $r_{i}$ denote the restrictions indicated by the diagram below; then for each

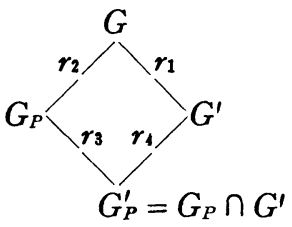

place $P$ in $S$ we have

$$
j_{P} \gamma_{4} \gamma_{1} \alpha_{2}=j_{P} \gamma_{3} r_{2} \alpha_{2}=r_{3} j_{P} r_{2} \alpha_{2}=r_{3} i_{P} \alpha_{P}=i_{P} r_{3} \alpha_{P}
$$

This shows that $\alpha_{2}^{\prime}=r_{1} \alpha_{2}$ as required, because $r_{3} \alpha_{P}$, the restriction of the local fundamental class for $G_{P}$, is the local fundamental class for $G_{P}^{\prime}$.

Let $N$ be a $Z$-module of finite rank $d$, on which $G$ operates, and put $M=$ $\operatorname{Hom}(N, Z)$. Then for every $G$-module $C$ we have a canonical isomorphism

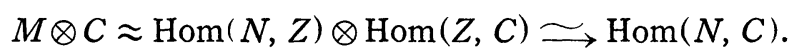

Thus, we can reinterpret the $G$-modules in $\left(1_{m}\right)$ as modules of homomorphisms rather than as tensor products in this case. In particular, we can interpret $\operatorname{Hom}(N, E)$ as a group of points on a certain group scheme. Let $R$ be the ring of elements of $K$ which are integers at all places $P$ not in $S$, and let $R_{0}=R \cap L=R^{G}$. Our conditions $(S 3)$ and (S4) on $S$ amount to saying that $R$ is etale over $R_{0}$, and that $R$ is a principal ideal ring. Let $A=R[N]$ be the group ring of $N$ over $R$, and let $A_{0}=A^{G}$, where $G$ operates on $A$ by the rule

$$
s\left(\sum_{x \in N} r_{x} x\right)=\sum_{x \in N}\left(s r_{x}\right)(s x)=\sum_{x \in N}\left(s r_{s-1}\right) x .
$$

Since $R$ is etale over $R_{0}$, the canonical homomorphisms

$$
A_{0} \underset{R_{0}}{\otimes} R \rightarrow A, \quad \text { and } \quad A_{0} \underset{R_{0}}{\otimes} A_{0} \rightarrow(A \underset{R}{\otimes} A)^{a}
$$

are isomorphisms. In virtue of the second of these, the homomorphism of $R$ algebras $A \rightarrow A \underset{R}{\otimes} A \approx R[N \times N]$ which is induced by the diagonal homomorphism of groups $N \rightarrow N \times N$ induces a homomorphism of $R_{0}$-algebras

$$
A_{0} \rightarrow A_{0} \underset{P_{0}}{\otimes} A_{0}
$$

This homomorphism gives a law of composition on the $R_{0}$-scheme $T=\operatorname{Spec}\left(A_{0}\right)$, by virtue of which $T$ becomes a commutative group scheme. We have, in 
view of the first of the isomorphisms (13),

$$
T \underset{R_{0}}{\times} R \approx \operatorname{Spec}(R[N]) \approx \mathbf{G}_{m, R}^{d},
$$

where $\mathbf{G}_{m, R}$ denotes the multiplicative group scheme over $R$, because

$$
R[N] \approx R\left[Z^{d}\right] \approx R[Z]^{\stackrel{\otimes}{R},},
$$

as soon as we forget the operation of $G$. Thus, $T$ is a "twisted torus of dimension $d$, defined over $R_{n}$, and split by the unramified extension $R^{\prime \prime}$. The group of points on $T$ rational over $R$ is

$$
T(R) \approx \operatorname{Hom}_{R \text {-algebras }}(R[N], R) \approx \operatorname{Hom}_{\text {Groups }}(N, E),
$$

because $E$ is the group of invertible elements in $R$. Thus the theorem above gives a description of the Galois cohomology groups of the torus $T$ for the extension $R / R_{0}$, in terms of the character module $N$ of $T$; from the point of view of algebraic groups, Nakayama's theorem [4] gives a standard procedure for generalizing theorems on the cohomology of $\mathbf{G}_{m}$ to arbitrary tori.

\section{BibLIOGRAPHY}

[1] E. Artin and J. Tate, Class Field Theory, Harvard, 1961.

[2] H. Cartan and S. Eilenberg, Homological Algebra, Princeton Math. Ser., 19, Princeton, 1956.

[ 3 ] G. Hochschild and T. Nakayama, Cohomology in class field theory, Ann. of Math., $\mathbf{5 5}$ (1952), pp. 348-366.

[4] T. Nakayama, Cohomology of class field theory and tensor product modules I, Ann. of Math., 65 (1957), pp. 255-267.

[ 5 ] J.-P. Serre, Corps locaux, Hermann, Paris, 1962.

[6] J. Tate, The higher dimensional cohomology groups of class field theory, Ann. of Math., 56 (1952), pp. 294-297.

[7] J. Tate, The cohomology groups of algebraic number fields, Proc. Int. Cong. Math., Amsterdam, Sept., 1954.

Harvard University 